\title{
Vorelsterzeitliche Karsttaschen mit Terra-fusca-Füllung in der Kreide von Meerdorf im Landkreis Braunschweig
}

\author{
Von Rudolf Herrmann, Hannover \\ Mit 4 Abbildungen
}

Zusammenfassung. Bei Meerdorf im Landkreis Braunschweig finden sich auf der Oberfläche von mergeligen Kalken der Mucronatenschichten sack- und trichterförmige Taschen, die bis $3 \mathrm{~m}$ tief und mit braunem Ton und Geschiebesanden gefüllt sind. Auf schwacher Hangneigung gelegene Taschen sind in ihrem oberen Teil hangabwärts verzerrt und unter Fließschutt der Kreidekalke zu langen Schwänzen ausgezogen. Der braune Ton ist auch außerhalb der Taschen auf der narbigen Oberfläche der Kalke verbreitet, wogegen die Geschiebesande auf die Kerne der Taschen beschränkt sind. Über diesen Bildungen liegt eine Grundmoräne.

Die Kleinformen der Kreideoberfläche und die tieferen Taschen sind durch Auflösung von Mergelkalkstein entstanden; der nichtkarbonatische Rückstand bildet eine Terra fusca. Die Verkarstung vollzog sich unter warmem Klima und begann möglicherweise schon vor dem Pleistozän. Die Geschiebesande in den Taschen sind vermutlich elsterzeitlich und waren ursprünglich flächenhaft verbreitet; in das Innere der Taschen sind sie durch Eiskeilbildung oder auf andere Weise gelangt. Danach wurden die Sande flächenhaft abgetragen. Deformiert wurden die Taschen zu Beginn der Saalezeit durch Solifluktion. Die das Profil abschließende Grundmoräne ist die des drenthestadialen Eisvorstoßes.

A bst ract. Near Meerdorf in the district of Braunschweig there are a kind of kettle- or funnel-shaped hollows or pockets at the surface of the marly chalk beds of Belemnitella mucronata. These pockets are of a depth of up to 3 metres and are filled with a brown clay and with glacial sands. Pockets that are to be found at slightly inclined slopes are deformed in their upper part in down-slope direction and are drawn out like long tails and they are covered by frost chalk debris. The brown clay is also spread at the scarred surface of the chalk outside the pockets, whereas the glacial sands are to be found exclusively in the interior of the pockets. The uppermost sediment is a basal moraine.

The scarred surface of the Cretaceous and the larger pockets originated in the dissolution of the marly chalk, the clay at its surface being the residues of the non-carbonates, a so-called Terra fusca. These processes of karstification came about under a warm climate. During a cold period, probably the Elster (Mindel) stage of the Glacial Period, the glacial sands have been deposited. Only the fractions of the sands that got into the pockets have remained. The deformation of the upper parts of the pockets is due to the solifluction that took place at the beginning of the Saale glacial stage. It was followed by the deposition of the basal moraine of the Drenthe stadial ice thrust.

Bei Meerdorf, etwa $15 \mathrm{~km}$ nordwestlich von Braunschweig, befinden sich an der Oberfläche mergeliger Kalke unter einer dünnen Moränendecke sack- und trichterförmige Kessel oder Taschen, die mit Ton und Glazialmaterial gefüllt sind; sie greifen knapp $3 \mathrm{~m}$ tief in das Gestein ein und sind - in Hanglage - in ihrem oberen Teil hangabwärts verzerrt. Diese in Kalkgruben aufgeschlossenen Taschen sind von anderen Bearbeitern schon beschrieben, aber in anderer Weise gedeutet worden, als im folgenden dargelegt wird.

Zuerst ist O. Sickenberg auf diese Aufschlüsse aufmerksam geworden. In einer im Jahre 1954 erschienenen Abhandlung sprach er sich dagegen aus, die Kessel als interglaziale "geologische Orgeln" aufzufassen, weil sie keine interglazialen Sedimente enthielten, und entschied sich dafür, sie als saaleeiszeitliche Gletschertöpfe mit Deformation durch das weiter vorrückende Eis zu deuten. Herr Sickenberg hatte die Freundlichkeit, mich schon vor Erscheinen seiner Abhandlung auf dieses bemerkenswerte geologische Objekt hinzuweisen. Bei Besuchen der Aufschlüsse kam ich zu der Auffassung, daß die Kessel das Ergebnis einer vor-saaleeiszeitlichen Karstverwitterung seien, der sie füllende Ton ein Rückstand der Kalkauflösung und die Deformation der Kessel ein Werk der Solifluktion sei. In einem Vortrag, den ich am 1.6.1955 in der 22. Jahresversammlung der Arbeitsgemeinschaft nordwestdeutscher Geologen in Braunschweig hielt, legte ich die Gründe für diese Auffassung dar. 
Neuerdings hat H. BRÜNING (1966) - ohne Kenntnis meines Vortrages - die Kessel als Periglazialerscheinungen gedeutet. Er bezeichnet sie ihrer Anlage nach als Frostkessel, in deren toniger Füllung sich Eiskeile entwickelten. Die Deformation der Kessel schreibt er ebenfalls der Solifluktion zu. O. Sickenberg stimmte nun dieser Periglazial-Hypothese bei.

Im folgenden wird dieser Hypothese die Deutung der Kessel als Karsttaschen gegenübergestellt und gezeigt, daß in den Meerdorfer Aufschlüssen ein komplexes Karst- und Periglazialprofil vorliegt. Der Aussagereichtum dieses Profils kann an dieser Stelle nicht ausgeschöpft werden. Die Darstellung beschränkt sich auf die Entwicklung der Karsttaschen, zieht aber auch das in deren Umgebung ausgebildete Bodenprofil heran, dessen abschließende Beurteilung einer bodenkundlichen Untersuchung überlassen bleibt. ${ }^{1}$ ) Es erscheint zweckmäßig, die hier mitzuteilenden Ergebnisse auf eine neue Beschreibung des Aufschlußbefundes aufzubauen, die zwar Wiederholungen früherer Aussagen einschließt, aber auch in mancherlei Hinsicht vervollständigt ist.

Die Mergelkalkgruben liegen $6 \mathrm{~km}$ nordöstlich von Peine beiderseits der von Meerdorf nach NW führenden Landstraße (Top. Karte 1:25000, Nr. 3627 Peine, Ausgabe 1955). Die Gruben sind in den Südostteil eines flach von 75 auf $85 \mathrm{~m}$ ü. N. N. ansteigenden Hügels eingeschnitten. Auf der Nordseite der Straße befindet sich auf $450 \mathrm{~m}$ Länge ein zusammenhängendes Grubengelände - im folgenden als nördliche Grube bezeichnet - mit einer 5 bis $6 \mathrm{~m}$ hohen Aufschlußwand. Die südliche Grube schneidet südlich der Straße von $\mathrm{S}$ her in den dort südwärts abfallenden Hang des Hügels $60 \mathrm{~m}$ breit ein und endet etwa halbkreisförmig mit einer 6 bis $7 \mathrm{~m}$ hohen Böschung.

Der Mergelkalkstein wird seit alter Zeit, aber jetzt abnehmend, zur Kalkung der Acker gewonnen. Vor 13 Jahren war das Anstehende an den Grubenwänden auf langen Strecken frisch angeschnitten; gegenwärtig - im Frühjahr 1968 - sind nur wenige Entnahmestellen offen; im übrigen sind die Wände verfallen. Die folgende Beschreibung stützt sich daher z. T. auch auf frühere Beobachtungen.

Die aufgeschlossenen Mergelkalksteine gehören den Unteren Mucronatenschichten an. Sie sind etwa in Dezimeterabständen geschichtet und stark geklüftet. In der Schichtenfolge wechseln weiße Kalksteine mit mürberen hellgrauen Mergeln. Die Nichtkarbonat-Gehalte dieser Gesteine betrugen in Einzelproben 12 bzw. 32\%. ${ }^{2}$ )

Die Schichtung läßt ein flaches Einfallen (etwa $5^{\circ}$ ) nach W bis WNW erkennen, wie es der Lage auf dem Ostflügel der Rosental-Meerdorfer Oberkreidemulde (WoLDSTEDT 1934) entspricht. Das Schichtenfallen bewirkt, daß in der nördlichen Grube insgesamt eine Schichtenfolge von etwa $50 \mathrm{~m}$ Mächtigkeit aufgeschlossen ist. Die Kalk- und Mergelkalkfazies ist in diesem Bereich einheitlich. - In der nördlichen Grube ist noch jetzt eine schon früher erkennbare westfallende Abschiebung aufgeschlossen; ihre Sprunghöhe ist wegen der Ahnlichkeit der beiderseits von ihr anstehenden Schichten nicht feststellbar.

In der obersten, bis zu 1,5 m Tiefe reichenden Zone ist das Gestein durch Zerfall in zentimetergroße Stückchen entschichtet. Dieser mechanische Gesteinszerfall ist schon von den früheren Beobachtern auf die Wirkung eiszeitlichen Frostes in der Frostwechselzone zurückgeführt worden.

Die Oberfläche des Frostschuttes ist narbig und zerfurcht und wird von einem braunen Ton bedeckt; dieser ist am Westrand der nördlichen Grube bis etwa $20 \mathrm{~cm}$ mächtig, keilt aber weiter östlich aus. In der südlichen Grube ist er nur schwach entwickelt. Außerhalb

1) Hinsichtlich der bodenkundlichen Probleme des Aufschlusses bin ich Herrn Dr. Roeschmann, Niedersächsisches Landesamt für Bodenforschung, Hannover, der die Aufschlüsse mit mir besuchte, für mannigfaltige Hinweise zu Dank verpflichtet.

2) Die analysierten Proben wurden am Weststoß der nördlichen Grube 10 bzw. $50 \mathrm{~cm}$ tief unter der Oberfläche der Mergelkalke entnommen. Für die Ausführung der Analysen bin ich Herrn Dr. FASTABEND, Bodenchemisches Labor des Niedersächsischen Landesamtes für Bodenforschung, sehr zu Dank verbunden. 


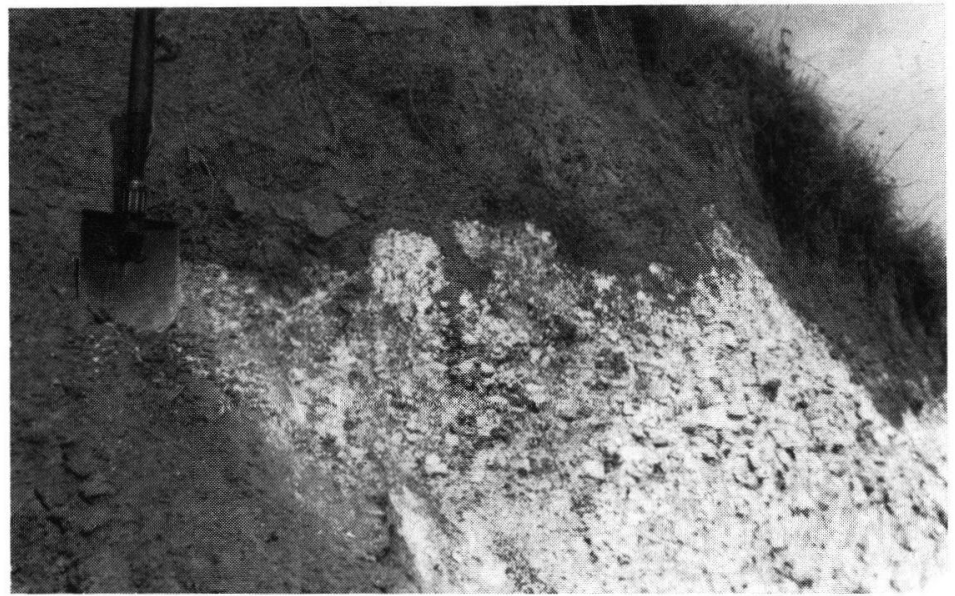

Abb. 1. Lösungsoberfläche des Kreidekalksteins mit zapfenförmig und adernetzartig eindringendem Ton der Terra fusca. Meerdorf, nördliche Grube, Weststoß, April 1968. 1 : 14.

der tonerfüllten Taschen greift der Ton zapfenförmig und adernetzartig in den Frostschutt ein (Abb. 1). Der Ton ist dicht, stark plastisch, leicht aufschlämmbar, schluffhaltig, kalkfrei, führt kleine Toneisensteingeoden, ist meist frei von anderen Einschlüssen und hat ein parallelepipedisches Gefüge.

Auf dem Ton liegt mit unscharfer Grenze ein sandiger Geschiebelehm, der etwa $1 / 4$ bis $1 / 2 \mathrm{~m}$ mächtig und meist gänzlich in der Ackerkrume aufgegangen ist. Stellenweise, so in der südlichen Grube, hat der Geschiebelehm Sand und Geschiebe an den liegenden Ton abgegeben, und es hat eine geringfügige Tonverlagerung zu diesem hin stattgefunden. Von dem Geschiebelehm aus greifen mit sandigem Lehm gefüllte Spalten durch den Ton hindurch bis in den Kalkstein-Frostschutt ein. Wo der Ton fehlt, liegt die geschiebeführende Ackerkrume unmittelbar auf dem Frostschutt.

Der umfassendste Einblick in die Erscheinungsweise der in den Mergelkalkstein eingreifenden Taschen ließ sich früher in der südlichen Grube gewinnen. An der Wand dieser Grube waren im Jahre 1955 auf 50 m Länge 19 Taschen sichtbar; jetzt liegen dort nur

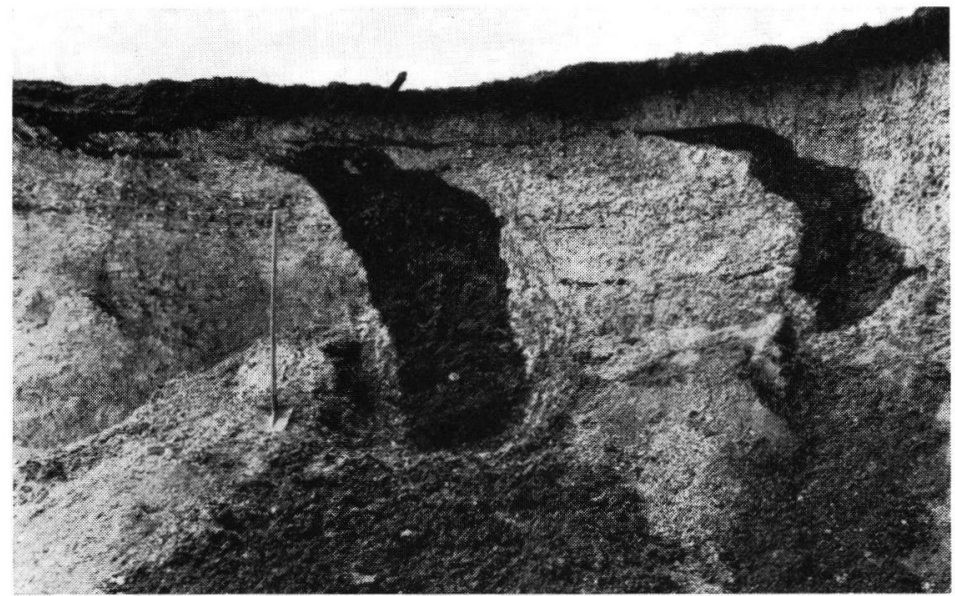

Abb. 2. Sackförmige Karsttaschen im Kreidekalkstein mit Ausschwänzung in der Solifluktionszone; Medianschnitt. Meerdorf, südliche Grube, Nordwestseite, April 1955. 1 : 80. 


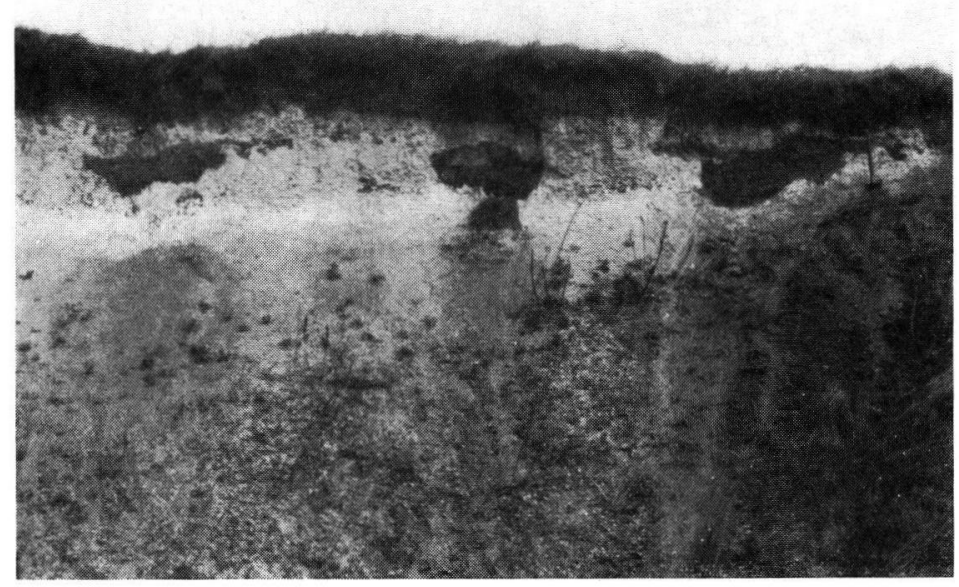

Abb. 3. Sackförmige Karsttaschen im Kreidekalkstein, quer geschnitten, mit beiderseitiger Ausschwänzung in der Solifluktionszone, darüber die saalezeitliche Grundmoräne mit (umgelagerter?) Terra fusca an der Basis. Meerdorf, südliche Grube, Nordseite, April 1968. 1 : 80.

sechs solcher Formen frei. Auf der halbkreisförmig verlaufenden Abschlußwand waren die Taschen je nach ihrer Lage in unterschiedlicher Weise angeschnitten. Im westlichen, etwa in der Richtung des Hanggefälles liegenden Teil der Aufschlußwand (der jetzt verfallen ist) zeigten die Taschen in Schnitten parallel zur Achse die Umrisse steilstehender Säcke mit hangabwärts gerichteter Verschleppung des oberen Teiles. Zwei nahe beieinander befindliche Taschen mit starker seitlicher Verschleppung ihrer oberen Teile wurden durch Aufgrabung im Vertikalschnitt freigelegt (Abb. 2). Mit ihrem tieferen, unverzerrten Teil reichten die Taschen etwa $3 \mathrm{~m}$ tief unter die Oberfläche; ihr Durchmesser bei rundlichem Querschnitt betrug etwa $1 \mathrm{~m}$, ihr Rauminhalt etwa 2 bis $2^{1 / 2} \mathrm{~m}^{3}$. Der untere Teil der Taschen griff in den geschichteten Mergelkalkstein ein, der obere Teil endete in der Frostschuttzone mit meterweit hangabwärts gezogenen Schwänzen.

Im mittleren und östlichen Teil der Aufschlußwand (der südlichen Grube) erscheinen die Taschen im Schnitt schiefwinklig zu ihrer Achse; liegt der Schnitt im verschleppten oberen Teil der Tasche, so ergibt sich ein rundlicher oder querovaler Umriß, wobei der Oberrand an einer oder beiden Seiten ausgeschwänzt sein kann (Abb. 3).

Der Ton, der dem Mergelkalkstein weithin aufliegt, kleidet auch die Taschen aus und umschließt darin einen sandigen Kern. Der Ton ist ockerbraun, partieweise auch (durch Humusgehalt?) dunkelbraun, im tieferen Teil der Taschen auch grau. In Taschenquerschnitten kommt eine konzentrische Anordnung von hellbraunem Ton (außen) und dunkelbraunem Ton (innen) vor. Der Ton ist in den trockenen Außenteilen der Taschenfüllungen wandparallel schalig abgesondert, und Schrumpfungsrisse zerteilen ihn in kleine parallelepipedische Stücke. Gelegentlich enthält der Ton Kalksteinbruchstücke aus dem Frostschutt. Nahe an den Taschen wird der Mergelkalkstein von Tonadern durchschwärmt.

Die Kernpartien der Taschenfüllungen sind uneinheitlich; Extreme sind geschiebefreie, überwiegend feine, braunrote Sande mit geringerer Verfestigung und gröbere, geschiebereiche Sande mit starker Verfestigung durch Eisenhydroxid. Große Geschiebe sind selten; kürzlich wurde ein rundliches Geschiebe eines dunkelgrauen kristallinen Quarzits von etwa $15 \mathrm{~cm}$ Durchmesser und daneben ein plattiges Stück eines hellroten feinkristallinen Granitgneises, etwa $20 \mathrm{~cm}$ lang und bis $5 \mathrm{~cm}$ dick, in Hochkantstellung angetroffen. In seiner Masse bleibt der sandige Kern schätzungsweise hinter der seiner Tonumkleidung 
zurück. Entsprechend der Gestalt der Taschen ist auch ihr sandiger Kern länglich-zapfenförmig.

In der hangabwärts gerichteten Verformung der Taschen wird der sandige Kern auch am Oberende von der Tonummantelung umschlossen; er hat dabei keinen Zusammenhang mit dem Geschiebelehm, der auf dem übergreifenden Kalkstein-Frostschutt liegt, und unterscheidet sich von diesem durch einen geringeren Gehalt an Feinmaterial.

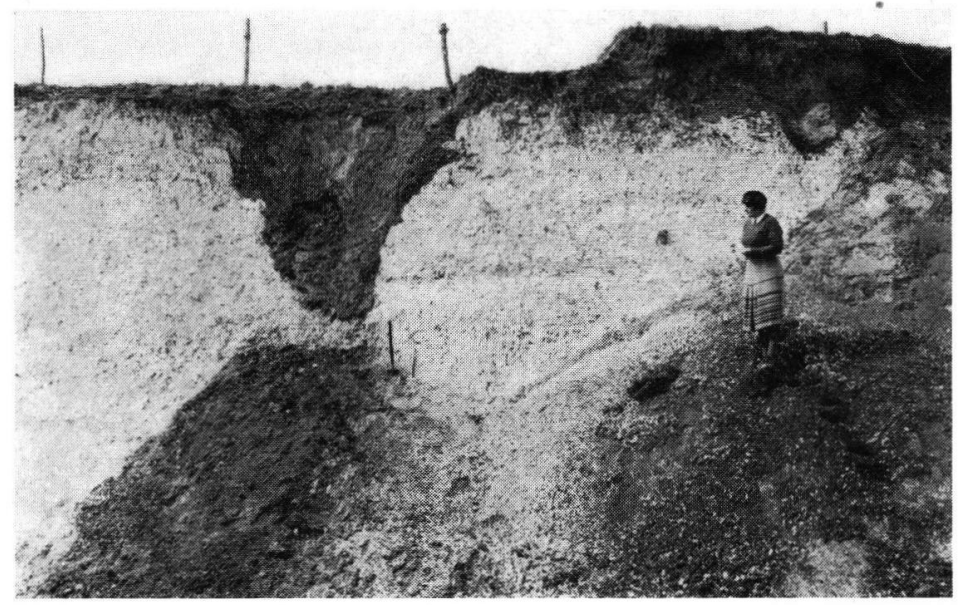

Abb. 4. Trichterförmige Karsttasche im Kreidekalkstein. Die Füllung ist größtenteils ausgeräumt; keine Deformation durch Solifluktion. Meerdorf, nördliche Grube, NW-Seite, April 1955. 1 : 90.

Im westlichen Teil der nördlichen Grube, der die flache Scheitelfläche des Hügels anschneidet, sind die Taschen trichterförmig (Abb.4) und haben ebenfalls eine Tonauskleidung und einen sandigen Kern, der sich mit der Grundmoräne darüber berührt. Weiter östlich war 1955 der hochovale Querschnitt einer Tasche angeschnitten, von deren Oberrand der Tonmantel an der Basis des Frostschuttes meterweit nach Osten - der Hangneigung entsprechend - ausgezogen war. Im östlichsten Teil der Grube sind z. Zt. einige kleine, nur etwa $1 / 2 \mathrm{~m}$ tiefe Trichter aufgeschlossen, offenbar die Spitzen größerer Trichter, deren oberer Teil abgetragen ist. Die Grundmoräne greift auch über diese Restformen hinweg.

In der ge netis chen De u tung des Aufschlußbildes stehen - entsprechend der Fragestellung - die Taschen mit ihrer Füllung im Vordergrund. Eine mechanische Ausräumung der $2 \mathrm{~m}^{3}$ und mehr Inhalt umfassenden Taschen ist nicht annehmbar, und wegen des Eingreifens der Taschen in den Dauerfrostboden kommt eine Wirkung von Kryoturbation nicht in Betracht. Die Taschen können nur durch Auflösung von Kalkstein entstanden sein; es sind $\mathrm{K}$ a r s t f o r m e n. Auch außerhalb der Karsttaschen hat der Kalkstein an seiner Oberfläche der Auflösung unterlegen. Die Lösungstätigkeit ist Sickerwässern zuzuschreiben, die auf einen tiefer liegenden Grundwasserspiegel absanken. Für die Lokalisierung der Taschen als Stellen bevorzugter Sickerbewegung sind keine Anzeichen erkennbar.

Gelbe Flecken im Kalkstein nahe unter seiner Oberfläche zeigen beginnende Entkalkung an. Der braune Ton auf der Kalkstein-Oberfläche und in den Taschen ist die aus der Auflösung der Karbonate hervorgehende Rückstandsbildung; er hat den Charakter einer Terra fusca. Die Menge der aufgelösten und weggeführten Karbonate beträgt, wie aus den Analysen zu schließen ist, ein Mehrfaches der Menge des tonigen Rückstandes. Die Füllung der Taschen mit Terra fuśca hat sich gleichzeitig mit ihrer langsamen Eintiefung vollzogen. Für die Umlagerung waren nur kurze Transporte aus der nächsten Umgebung erforderlich. 
Die Terra fusca von Meerdorf ist, wie die anderer Vorkommen in Mitteleuropa, fossil (MüCKenHAusen 1962). Für die Zeit ihrer Entstehung ist ein warmes Klima anzunehmen. Die früher in den Taschen vermißten Sedimente einer Warmzeit sind in der Terra fusca gegeben.

Es ist nicht unwahrscheinlich, daß die Bildung der Terra fusca frühzeitig - vielleicht schon vor dem Pleistozän - begonnen und sich in den Warmzeiten des Pleistozäns fortgesetzt hat. Mit der Füllung der Taschen war sie im wesentlichen beendet. Während der Kaltzeiten des Pleistozäns war sie unterbrochen und wurde durch Frostwirkung in und unter der Terra fusca abgelöst.

Die Geschiebesande im Kern der Taschen stammen aus einer Ablagerung, die auf der Terra fusca flächenhaft verbreitet war. Es waren Sedimente einer Vereisung, entweder von Schmelzwässern im Vorland eines Inlandeises abgesetzt oder aus einer Grundmoräne ausgewaschen. In das Innere der Taschen können die Geschiebesande als Ausfüllungen von Eiskeilspalten gelangt sein, wie schon H. BRüNING annahm. Die Eiskeile würden als spitze Einstiche in den Tonfüllungen entstanden sein; sie hätten auch die noch darüber verbreiteten Geschiebesande durchsetzt, aus denen sie ihre Füllung erhielten. Es sei dahingestellt, ob das Lageverhältnis zwischen Tonmantel und Sandkern in den Taschen auch als Ergebnis von Kryoturbation aufgefaßt werden kann. In jedem Fall sind die Sandkerne in den Taschen unter kaltem Klima entstanden.

Für die Altersstellung dieser Kaltzeit kommt in erster Linie die Elsterzeit in Betracht, deren Inlandeis südwärts weit über die Aufschlußörtlichkeit hinaus vorgedrungen war. Für die Karsttaschen folgt daraus ein vorelsterzeitliches Alter. Eine Bestätigung dieser Annahme durch eine Geschiebezählung war bisher aus Mangel an einer ausreichenden Zahl von Geschieben aus den Taschen nicht möglich.

Es folgt die Abtragung der Geschiebesande, frühestens noch am Ausgang der Elsterzeit oder in der Holsteinzeit.

Für die Solifluktion, die den Boden bis hinab in den Frostschutt in Bewegung setzte und die Taschen deformierte, ergibt sich ein frühsaalezeitliches Alter. Die über alles hinweggreifende Grundmoräne ist die des Drenthestadiums der Saaleeiszeit. (Die geringmächtige, geschiebeführende Terra fusca an deren Basis in der südlichen Grube ist wohl umgelagert und nicht an dieser Stelle neu - eemzeitlich — entstanden.)

Es hat sich also folgende Entwicklung ergeben:

1. (Mehrfacher?) Wechsel von Karstverwitterung und Terra-fusca-Bildung unter warmem Klima (vorelsterzeitlich) und Frostwirkung unter kaltem Klima (Elsterzeit, auch ältere Kaltzeiten ?).

2. Überlagerung der Terra fusca mit Geschiebesand in der Elsterzeit.

3. Eiskeilbildung (oder Kryoturbation?) in den Karsttaschen und Füllung mit Geschiebesand im Ausgang der Elsterzeit.

4. Flächenhafte Abtragung der Geschiebesande, noch Elsterzeit bzw. Holsteinzeit.

5. Solifluktion mit Deformation der Taschen, sofern sie auf geneigtem Hang lagen, zu Beginn der Saaleeiszeit.

6. Überlagerung mit Grundmoräne des Drenthestadiums.

(Stellt man die Geschiebesande zu einem frühsaalezeitlichen Eisvorstoß - Drenthe I -, so reicht die Terra-fusca-Bildung bis in die Holsteinzeit, und es ergeben sich weitere zeitliche Verschiebungen, von deren Aufzählung abgesehen werden kann.)

Schließlich sei noch auf die Aussage hingewiesen, die die Meerdorfer Aufschlüsse zur Frage der Hangentwicklung im Flachland beitragen. Verkarstung, glaziale Sedimentation, Solifluktion und Abtragung haben die Hänge des Hügels, an dessen Großformen gemessen, jeweils nur um geringe Beträge verändert.

Lösungstaschen und ähnliche Formen sind in Karstgebieten weit verbreitet und sind verschiedentlich beschrieben worden. Im $\mathrm{n}$ ord de u $\mathrm{ts}$ che $\mathrm{K}$ reidegebiet stellen 
die Vorkommen von Lägerdorf, Rügen und Meerdorf und das von Maastricht weit auseinander liegende Punkte ihrer Verbreitung dar.

Die Karstformen an der Oberfläche der Lägerdorfer Kreide, die E. M. TodtmanN (1951) beschrieben hat, weisen Übereinstimmungen mit denen von Meerdorf auf; aber sie sind formenreicher und größer dimensioniert; die Bildung von Rückstandston - als grauer Ton mit Feuerstein - tritt bei nur 1,4\% Nichtkarbonat-Gehalt in der Kreide von Lägerdorf im Vergleich zu Meerdorf erheblich zurück. Aus der Einsenkung von saalezeitlichem Geschiebemergel in die Vertiefungen der Kreideoberfläche, auch in Taschen mit Tiefen in der Größenordnung von $10 \mathrm{~m}$, ergibt sich deren Datierung als nachsaalezeitlich. Solifluktionserscheinungen sind nicht beschrieben worden.

Von der Schreibkreide von Rügen hat A. LUDwIG (1954) unter Grundmoräne liegende geologische Orgeln beschrieben, die mit Verwitterungslehm ausgekleidet sind und einen sandigen Kern haben. Die Verkarstung war nach der dafür gegebenen Erklärung mit einer Tonauswaschung aus der Grundmoräne und Anreicherung des Tons an der Grenze gegen die Kreide verbunden. $\mathrm{Da}$ es sich um jüngste Grundmoräne handelt, sind Verwitterung und Orgelbildung postglazial.

Bei Maastricht werden die Kreideschichten des Petersberges nach der Beschreibung von Mathieu (1814), der dort zuerst von einer „Geologischen Orgel“ sprach, von natürlichen Schächten oder Röhren durchsetzt, deren Durchmesser $2^{1 / 2} \mathrm{~m}$ und deren Länge Zehner von Metern erreichen kann. Die Schächte sind ursprünglich mit Lockermassen erfüllt, sind aber in verschiedener Höhe des Berges durch Steinbruchstollen angeschnitten und teilweise gegen diese hin entleert. Sie befinden sich „an einigen Stellen so dicht beieinander, daß sie sich fast berühren“, und bilden in dieser Gruppierung eine „geologische Orgel“, wobei die einzelne Schachtröhre mit einer Orgelpfeife zu vergleichen ist.

Die Dimensionen der geologischen Orgeln des Petersberges werden in den genannten anderen Vorkommen bei weitem nicht erreicht. In Meerdorf können die Taschen nur als Anfangsstadien von Schächten angesehen werden, zu deren Vertiefung es unter den gegebenen örtlichen Verhältnissen nicht gekommen ist.

\section{Schriften}

BrüNiNG, H.: Vorkommen und Entwicklungsrhythmus oberpleistozäner Periglazial-Erscheinungen und ihr Wert für pleistozäne Hangformung. Forsch. dtsch. Landesk. 156, 100 S., 52 Abb. auf Taf., Bad Godesberg 1966.

Herrmann, R.: Ein vorsaaleeiszeitliches Verwitterungsprofil auf Kreidemergel in der Umgebung von Braunschweig. Vortrag, gehalten am 1.6. 1955 in Braunschweig in der 22. Jahresversammlung der Arbeitsgemeinschaft nordwestdeutscher Geologen (unveröffentlicht).

Ludwig, A.: Geologische Orgeln in der Schreibkreide Rügens. Wiss. Z. E. M. Arndt-Univ. Greifswald 4, math.-naturw. Reihe, Nr. 3/4, 1954/55.

Mathiev: Entdeckung natürlicher Schächte oder Röhren, welche die Kalksteinbänke des Petersberges bei Maastricht durchsetzen. - Journ. Mines 5, 34 (Auszug von L. W. GiLbert, Ann. Phys. 46, 402-405, Leipzig 1814).

Mückenhausen, E.: Entstehung, Eigenschaften und Systematik der Böden der Bundesrepublik Deutschland. 148 S., 14 Abb., 60 Taf., Frankfurt/M. (DLG-Verl.) 1962.

Sickenberg, O.: Gletschertöpfe aus der Saaleeiszeit im Harzvorland. Neues Arch. Niedersachsen, Jg. 1954, 61-65, 10 Abb. auf Taf., Hannover 1954.

Todtmann, E. M.: Unterirdischer Karst in der Kreide von Lägerdorf bei Itzehoe. Schr. naturw. Ver. Schlesw.-Holstein 25 (Karl-Gripp-Festschr.), 125-130, 7 Abb., Kiel 1951.

Woldstedt, P.: Über den Charakter schmaler Störungszonen und breiter Sättel im Gebiet zwischen Harz und Hannover. Jb. preuß. geol. Landesanst. f. 1934, 55, 93-102, 2 Abb., 1 Taf., Berlin 1934.

Manuskr. eingeg. 15. 5. 1968.

Anschrift des Verf.: Dr. Rudolf Herrmann, Niedersächsisches Landesamt für Bodenforschung, 3 Hannover-Buchholz, Alfred-Bentz-Haus. 